\title{
Load Balanced and Optimal Disk Allocation Strategy for Partial Match Queries on Multidimensional Files
}

\author{
Sajal K. Das, Member, IEEE, and Cristina M. Pinotti, Member, IEEE Computer Society
}

\begin{abstract}
A multidimensional file is one whose data are characterized by several attributes, each specified in a given domain. A partial match query on a multidimensional file extracts all data whose attributes match the values of one or more attributes specified in the query. The disk allocation problem of a multidimensional file $F$ on a database system with multiple disks accessible in parallel is the problem of distributing $F$ among the disks such that the data qualifying for each partial match query are distributed as evenly as possible among the disks of the system. We propose an optimal solution to this problem for multidimensional files with pairwise prime domains based on a large and flexible class of maximum distance separable codes, namely, the redundant residue codes. We also introduce a new family of residue codes, called the redundant nonpairwise prime residue codes, to deal with files whose attribute domains are nonpairwise prime.
\end{abstract}

Index Terms-Nonuniform multidimensional file, partial match query, strictly optimal disk allocation, redundant pairwise-prime residue code, redundant nonpairwise prime residue code.

\section{INTRODUCTION}

Darallel database systems are essential to important 1 real-life applications that require to manage extremely large volumes of data, often of the order of terabytes, which cannot be stored in a single disk. The applications include spatial databases, airline reservation systems, cartography, World Wide Web, etc. Queries on such large databases involve the retrieval of a big portion of data, often under critical time constraints. Therefore, to retrieve qualifying data in parallel is mandatory. Before proceeding further, let us define a few terminology and notations.

In this paper, we consider database systems of multidimensional files, each made up of buckets, each, in turn, made up of records. A record is an $n$-tuple $\left\langle r_{1}, \ldots, r_{n}\right\rangle$ of attributes such that the value of the $j$ th attribute $r_{j}$ belongs to the interval $I_{j}=\left[L_{j}, R_{j}\right]$, for $1 \leq j \leq n$.

In order to define a bucket, let us uniformly partition the values of the $j$ th attribute of a record into $m_{j}$ groups, each of size

$$
\left\lceil\frac{R_{j}-L_{j}+1}{m_{j}}\right\rceil,
$$

and let $m_{j}$ be termed the domain of $r_{j}$. The bucket < $x_{1}, \ldots, x_{n}>$ of respective domains $m_{1}, \ldots, m_{n}$ is the set of the records $\left\langle r_{1}, \ldots, r_{n}\right\rangle$ such that the value of $r_{j}$, for $1 \leq j \leq n$, belongs to the $x_{j}$ th group of $I_{j}$. More formally,

- S.K. Das is with the Department of Computer Science and Engineering, University of Texas at Arlington, Arlington, TX 76019-0015.

E-mail: das@cse.uta.edu.

- C.M. Pinotti is with the Department of Computer Science and Telecommunications, University of Trento, Povo 38050, Italy.

E-mail: pinotti@science.unitn.it.

Manuscript received 21 Aug. 2001; revised 8 Apr. 2002; accepted 22 Apr. 2002.

For information on obtaining reprints of this article, please send e-mail to: tpds@computer.org, and reference IEEECS Log Number 114821.

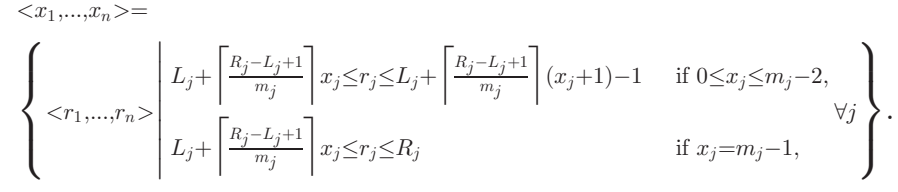

Thus, the bucket $\left\langle x_{1}, \ldots, x_{n}\right\rangle$ stores at most

$$
\Pi_{j=1}^{n}\left\lceil\frac{R_{j}-L_{j}+1}{m_{j}}\right\rceil
$$

records. Finally, a multidimensional file $F\left(m_{1}, \ldots, m_{n}\right)$ of domains $m_{1}, \ldots, m_{n}$ is the set of all $\Pi_{i=1}^{n} m_{i}$ buckets $\left.<x_{1}, \ldots, x_{n}\right\rangle$ where $x_{i} \in\left[0, \ldots, m_{i}-1\right]$ and $1 \leq i \leq n$.

Example 1. Consider two attributes that range, respectively, in $I_{1}=[1 \ldots 100]$ and $I_{2}=[1 \ldots 50]$. Fixing the domains $m_{1}=20$ and $m_{2}=10$, partition $I_{1}$ and $I_{2}$, respectively, into 20 and 10 groups of equal size. Specifically, the $i$ th group of $I_{1}$ contains the values $[5 i+1,5 i+2$, $5 i+3,5 i+4,5(i+1)]$, for $0 \leq i \leq 19$, of the first attribute. Thus, the bucket $<2,3>$ contains the records $\left\langle r_{1}, r_{2}\right\rangle$, where $11 \leq r_{1} \leq 15$ and $16 \leq r_{2} \leq 20$. The multidimensional file $F(20,10)$ consists of all the 200 buckets $\left.<x_{1}, x_{2}\right\rangle$, where $0 \leq x_{1} \leq 19$ and $0 \leq x_{2} \leq 9$, and each bucket stores 25 records.

A uniform p-ary multidimensional file $F(p, \ldots, p)$ is defined as one in which all the domains assume the identical value $p$. Otherwise it is called a nonuniform multidimensional file. If the $n$ domains $m_{1}, \ldots, m_{n}$ are pairwise prime, $F\left(m_{1}, \ldots, m_{n}\right)$ is said to be a nonuniform, pairwise prime multidimensional file.

The most common operation on a database system is the information retrieval. Formally,

Definition 1. Given a multidimensional file $F\left(m_{1}, \ldots, m_{n}\right)$, a match query $q=<q_{1}, \ldots, q_{n}>$ on $F$ retrieves the bucket 
$<q_{1}, \ldots, q_{n}>$. A partial match query (PMQ) is one whose $i$ th attribute $q_{i}$ is either specified or unspecified, and retrieves from $F$ the set of buckets that match the query $q$ on the specified attributes. Such buckets are said to qualify for q. The query response set, $Q R(q)$, of a PMQ with the unspecified attributes $q_{i_{1}}, q_{i_{2}}, \ldots, q_{i_{k}}$ consists of $N(q)=$ $\Pi_{j=1}^{k} m_{i_{j}}$ buckets that qualify for $q$.

As an example, given the file $F(20,10)$, the PMQ $q=$ $<5, *>$ extracts $N(<5, *>)=10$ buckets defined as $\{<5, i>\mid 0 \leq i \leq 9\}$.

In real database systems, however, the number of attributes is much larger than two. Typically, there are thousands of buckets, and a considerably large number of them may qualify for each PMQ. In such a context, database systems need to store their files on multiple disks.

A parallel database system is a database system that stores multidimensional files on multiple disks that can be read or written simultaneously. This additional capability may improve significantly the time required for data transmission if a suitable scheme is adopted for data distribution among the disks. In other words, if the data that qualify for a query are almost evenly distributed among the disks available in the database system, the response time is reduced by a factor equal to the number of disks. In fact, distributing $F\left(m_{1}, \ldots, m_{n}\right)$ into a parallel database system of $D$ disks, denoted as $d_{0}, \ldots, d_{D-1}$, the time to retrieve the query response set $Q R(q)$ of a partial match query $q$ is proportional to the number of buckets that qualify for $q$ in each disk. Precisely, the response time of a query is proportional to $R T(q)=\max _{0 \leq i \leq D-1}\left\{N_{i}(q)\right\}$, where $N_{i}(q)$ is the number of buckets that qualify for $q$ stored on disk $d_{i}$. Thus, the response time is minimum when

$$
R T(q)=\left\lceil\frac{N(q)}{D}\right\rceil,
$$

i.e., when the query response set $Q R(q)$ is balanced among all the available disks in the system. Therefore, an optimal solution to the disk allocation problem is equivalent to finding a load balanced distribution of the buckets among the multiple disks of the system.

We adopt the following criteria for optimality.

Definition 2. [1], [7]. Given a system with $D$ disks that can be accessed simultaneously, a disk allocation strategy is termed optimal for a specific partial match query $q$ if it evenly distributes $N(q)$ among the $D$ disks, thus achieving a response time of $\left\lceil\frac{N(q)}{D}\right\rceil$ for $q$. Moreover, a disk allocation strategy is called strictly optimal if it is optimal for all partial match queries.

From now on, whenever the domains of the attributes are clear from the context, $F\left(m_{1}, \ldots, m_{n}\right)$ will be referred to as simply $F$.

\section{Previous Work on the Disk Allocation Problem}

The problem of minimizing the response time of PMQs on uniform multidimensional files has received a lot of attention in the past. For example, one of the first approaches to the problem proposed of distributing $F$ among $D$ disks with the help of a (pseudo) random number generator, with $1 / D$ as the probability of assigning a bucket to a particular disk. This solution had no constraints on the number of disks as well as on the cardinality of the domains of the attributes. However, it did not guarantee optimality for any class of partial match queries.

Subsequently, Fang et al. [6] interpreted each bucket of $F\left(m_{1}, \ldots, m_{n}\right)$ as a point of the $n$-dimensional space $m_{1} \times \ldots \times m_{n}$, and suggested to partition the buckets into two geometrical similar groups; that is, in two groups with almost the same spanning tree and almost the same set of short spanning paths. They proved that, as a consequence of the geometrical similarity in each group, almost the same number of buckets qualifies for each PMQ. This solution, however, can at most halve the query response time. Moreover, the partitioning of $F$ in more than two groups seems quite intricate and, therefore, such a method is not suitable for generalizations.

A different approach, due to Du and Sobolewski [5], assumed a $D$-disk system and assigned the bucket

$$
<x_{1}, \ldots, x_{n}>
$$

to the disk $d_{j}$, where $j \equiv\left(\sum_{i=1}^{n} x_{i}\right) \bmod D$. This method has been shown to be always strictly optimal for PMQs with exactly one unspecified attribute. Moreover, for PMQs with two unspecified attributes, the method is strictly optimal when the disk system as $D=2$ or $D=3$, or when the attribute domains satisfy $m_{i} \equiv 0$ or $1 \bmod D$, for $1 \leq i \leq n$.

Later, Kim and Pramanik [8] solved the disk allocation problem optimally for both PMQs with exactly two unspecified attributes and for PMQs with the domain of one unspecified attribute larger than $D$. Their solution assigns the bucket $\left\langle x_{1}, \ldots, x_{n}\right\rangle$ to the disk $d_{j}$, such that $j=\left([+]_{i=1}^{n} x_{i}\right) \bmod D$, where $[+]$ denote the bitwise Exclusive-OR operation.

In [7], Faloutsos and Metaxas followed a completely different approach. Given a binary multidimensional file $F$ with $n$-attributes and $D=2^{k}$ disks, their solution assigns to each disk a set of buckets which form a linear $(k-1)$-error correcting binary code $C$. The code definition implies that any two buckets stored in the same disk differ by at least $k$ attributes. This approach is always optimal for PMQs with at most $(k-1)$ unspecified attributes and strictly optimal when $C$ is a maximum distance separable (MDS) code [10]. Unfortunately, for many pair of values $n, k$, there are no MDS codes; so, rarely, a strictly optimal solution can be found.

Abdel-Ghaffar and El Abbadi [1] extended the results of [7] to uniform $p$-ary multidimensional files, with $p>2$, and formally established the equivalence between strictly optimal allocation strategies and maximum distance separable codes. Based on such an equivalence and using the fact that the Reed-Solomon codes form a large family of $p$-ary MDS codes applicable to $p$-ary multidimensional files with at most $p-1$ attributes, the designed strategy is strictly optimal for files with at most $p^{p-1}$ buckets and uses $D=p^{t}$ disks, where 
$t \leq p-1$. This solution does not work for nonuniform files as well as for files whose records have more than $p-1$ attributes.

\section{Our Contributions}

This paper presents the first systematic solution to the disk allocation problem for nonuniform pairwise-prime multidimensional files. It can be considered as an extension of the results in [7], [1]. Our method takes full advantage of all (possibly a very large number) disks and, hence, the response time is drastically reduced. The solution is based on a very large and flexible class of semilinear MDS codes, called the redundant residue (pairwise prime) codes [2]. The existence of such a family of codes guarantees strictly optimal solution and alleviates the limitations of the previous code-based disk allocation strategies which mainly suffer from the lack of enough MDS codes for files of different sizes. Our solution handles nonuniform multidimensional files that model real data much better than the uniform multidimensional files, as argued below with an example.

Example 2. Let us consider a multidimensional file $F$ which has three integer attributes whose intervals are very skewed. Let the interval of the first attribute be $I_{1}=[1 . .1000]$, that of the second be $I_{2}=[1 . .21]$, and that of the third be $I_{3}=[1 . .9]$. Thus, there are $1000 \times$ $21 \times 9$ records altogether. Assuming that each disk page contains no more than 100 records, let us determine a suitable domain for each attribute such that a bucket has almost the same size as a disk page and each bucket is full.

First, we consider the scenario when all the three attributes have the same domain value $m_{1}=m_{2}=$ $m_{3}=p$. Then, the bucket $\left\langle x_{1}, x_{2}, x_{3}\right\rangle$, with $0 \leq x_{i} \leq$ $p-1$ for $1 \leq i \leq 3$, contains the records $<r_{1}, r_{2}, r_{3}>$ such that

$L_{i}+\left\lceil\frac{R_{i}-L_{i}+1}{p}\right\rceil x_{i} \leq r_{i} \leq L_{i}+\left\lceil\frac{R_{i}-L_{i}+1}{p}\right\rceil\left(x_{i}+1\right)-1$. Specifically,

- For $p=4$, we obtain very large buckets that do not fit in one disk page. Indeed, there are $250 \times$ $6 \times 3$ records stored in each bucket.

- For $p=10$, each bucket consists of $100 \times 3 \times 1$ records, which is still too large in size.

- For $p=20$, each bucket has at most $50 \times 2 \times 1$ records. Although a bucket perfectly fits in a page, the file $F$ is mapped into $20^{3}$ buckets, most of which are empty (consider, for example, all the buckets with $x_{3} \geq 1$ ).

On the other hand, if different domains are allowed a much better load factor can be obtained:

- For $m_{1}=20, m_{2}=11$ and $m_{3}=9$, the bucket $<x_{1}, x_{2}, x_{3}>$, for $0 \leq x_{1} \leq 19,0 \leq x_{2} \leq 10$ and $0 \leq x_{3} \leq 8$, consists of the records $<r_{1}, r_{2}, r_{3}>$ such that

$$
\begin{aligned}
& 1+x_{1}\left\lceil\frac{1000}{20}\right\rceil \leq r_{1} \leq\left(x_{1}+1\right)\left\lceil\frac{1000}{20}\right\rceil, \\
& 1+x_{2}\left\lceil\frac{21}{11}\right\rceil \leq r_{2} \leq \min \left\{\left(x_{2}+1\right)\left\lceil\frac{21}{11}\right\rceil, 21\right\}, \text { and } \\
& 1+x_{3} \leq r_{3} \leq\left(x_{3}+1\right) .
\end{aligned}
$$

Hence, each bucket $<x_{1}, x_{2}, x_{3}>$ consists at most of 100 records and the file $F$ has $20 \times 11 \times 9=$ 1,980 buckets, all of which are full.

This example shows that partitioning all attributes of the file with the same domain, one can get many empty buckets. On the contrary, if different domains are used for different attributes, a good load factor of the buckets can be obtained.

Back to the proposed disk allocation strategy: Note that the family of redundant residue codes applies only to files whose attributes have pairwise-prime domains. It is worth to point out that this is not a serious limitation since the attribute domains are selected when the disk allocation strategy is designed. For the sake of completeness, however, we introduce a new class of Redundant Nonpairwise Prime Residue Codes in order to handle attributes with nonpairwise prime domains. This may lead to a slowdown with respect to the optimal solution by a predetermined factor.

The remainder of the paper is organized as follows: Section 4 reviews basic concepts of the coding theory that are relevant to our work. Based on the redundant pairwise prime residue codes, Section 5 proposes a strictly optimal disk allocation strategy for nonuniform multidimensional files whose attributes have pairwise-prime domains. In Section 5.1, we study how to guarantee strict optimality when either the number of attributes of the file or the number of disks in the system changes. Finally, a new family of redundant residue codes, called the redundant nompairwise prime residue codes, is introduced in Section 6 to solve the disk allocation strategy for nonuniform multidimensional files whose attributes have nonpairwise prime domains. Finally, conclusions are offered in Section 7.

\section{Coding Theory Framework}

Given a set of $n$ positive integer radices, denoted as $m_{1}, m_{2}, \ldots, m_{n}$, let $S=m_{1} \times m_{2} \times \ldots \times m_{n}$ be the space of all $n$-tuples of size $M=\prod_{i=1}^{n} m_{i}$. A code $C$ is a subset of $S$. Each $n$-tuple of $S$ which is also in $C$ is called a codeword. Since $S$ is defined on $n$ radices, $C$ has length $n$. Let the size $\gamma$ of $C$ be its cardinality. If all radices are equal to $p \geq 2, C$ is called a $p$-ary code.

A code $C$ is linear if it is closed under the addition and subtraction operations, as defined below. Given two codewords $x=<x_{1}, x_{2}, \ldots, x_{n}>$ and $y=<y_{1}, y_{2}, \ldots, y_{n}>$ of $C$, both the $n$-tuples $x+y$ and $x-y$ as defined below are also codewords of $C$.

$$
\begin{aligned}
x+y=< & \left(x_{1}+y_{1}\right) \bmod m_{1},\left(x_{2}+y_{2}\right) \\
& \bmod m_{2}, \ldots,\left(x_{n}+y_{n}\right) \bmod m_{n}>, \text { and } \\
x-y=< & \left(x_{1}-y_{1}\right) \bmod m_{1},\left(x_{2}-y_{2}\right) \\
& \bmod m_{2}, \ldots,\left(x_{n}-y_{n}\right) \bmod m_{n}>.
\end{aligned}
$$


Let the information set $I_{S_{k}}=\left\{m_{i_{1}}, m_{i_{2}}, \ldots, m_{i_{k}}\right\}$ of a code $C$ be a set of $k$ indices such that for any $k$-tuple $a_{i_{1}}, a_{i_{2}}, \ldots, a_{i_{k}}$, where $a_{i_{j}} \in\left[0, m_{i_{j}}-1\right]$, there is a unique codeword $\left\langle x_{1}, x_{2}, \ldots, x_{n}\right\rangle \in C$ such that $x_{i_{j}}=a_{i_{j}}$ for $1 \leq j \leq k$. Hence, if a code $C$ has an information set $I_{S_{k}}$, then the size of $C$ is larger than or equal to $\gamma=\prod_{j=1}^{k} m_{i_{j}}$. Such a code $C$ is said to be a systematic code.

For a linear code $C$, the Hamming weight of a codeword $x=<x_{1}, x_{2}, \ldots, x_{n}>$ is the number of nonzero components $x_{i}$, and the Hamming distance between two codewords is the number of components in which they differ. The minimum distance $d$ of $C$ is the minimum Hamming distance between all pairs of distinct codewords in $C$. Since the all-zero codeword always belong to a linear code, the minimum distance $d>0$ and the minimum Hamming weight of the codewords of $C$ coincide.

The concept of minimum distance is essential for defining the error control capabilities of a code. In fact, representing the error as an $n$-tuple $e=<e_{1}, e_{2}, \ldots, e_{n}>$ and denoting a codeword $x$ subject to error $e$ as $x+e$, a code of minimum distance $d$ is able 1) to detect at least all errors $e$ with Hamming weight at most $d-1$ and 2) to correct all errors $e$ with Hamming weight $\leq\lfloor(d-1) / 2\rfloor$. It follows directly that, among all the codes of a given length $n$ and size $\gamma$, the codes with the highest error control are those with the largest minimum distance $d$.

From now on, $C=[n, \gamma, d]$ will denote a linear code of length $n$, size $\gamma$ and minimum distance $d$. Furthermore, when $\max \left\{\Pi_{i=1}^{k} m_{j_{i}}\right\} \leq \gamma<\max \left\{\Pi_{i=1}^{k+1} m_{j_{i}}\right\}$, the inequality $d \leq n-k+1$ holds, which is known as the Singleton bound [10]. The class of codes that satisfy the equality $d=$ $n-k+1$ plays an important role in our solution approach as evident from the following definition.

Definition 3. [10] A code $C=[n, \gamma, d]$, where $\gamma$ is the product of any $k$ radices, with minimum distance $d=n-k+1$ is called a maximum distance separable (MDS) code which is also a systematic code. Any $k$ radices of an MDS code $C$ form an information set.

Among the MDS codes, of particular interest are the Redundant Pairwise-Prime Residue Codes, which are defined on a Redundant Residue Number System (RRNS). An RRNS has $n$ pairwise-prime positive radices,

$$
m_{1}, m_{2}, \ldots, m_{k}, m_{k+1}, \ldots, m_{n},
$$

called moduli. Let the first $k$ moduli be termed as the nonredundant moduli, while the remaining $n-k$ moduli be the redundant moduli. Let $M_{I_{S}}=\Pi_{i=1}^{k} m_{i}$ and $M_{R}=$ $\Pi_{i=k+1}^{n} m_{i}$ be the product of, respectively, the nonredundant and the redundant moduli. Also, let $M=\prod_{i=1}^{n} m_{i}$ be the product of all moduli.

It is well known that every integer $X \in[0, M)$ can be uniquely represented by a residue vector

$$
x=<x_{1}, x_{2}, \ldots, x_{n}>
$$

such that $x_{i} \equiv X \bmod m_{i}$, for $1 \leq i \leq n$. Clearly, $0 \leq x_{i}<m_{i}$. Similarly, given a residue vector $x$, the corresponding integer $X$ can be uniquely determined by applying the Chinese Remainder Theorem [2]. The residue representations of the integers in the range $[0, M)$ can be partitioned into codes as follows.
Definition 4. For fixed $k \in[1, \ldots, n-1]$ and given a set of $n$ pairwise-prime redundant moduli,

$$
m_{1}, m_{2}, \ldots, m_{k}, m_{k+1}, \ldots, m_{n},
$$

let $M_{I_{S}}=\Pi_{i=1}^{k} m_{i}, M_{R}=\Pi_{i=k+1}^{n} m_{i}$, and $M=\Pi_{i=1}^{n} m_{i}$. For fixed $\alpha \in\left[0, M-M_{I_{S}}\right)$, the redundant residue $R R$ $(n, k, \alpha)$-code (or briefly, $R R$ - $(n, k)$-code) consists of all the residue vectors representing integers in the range $\left[\alpha, \alpha+M_{I_{S}}\right)$. In particular, for $\alpha=0, R R$ - $(n, k, 0)$-code consists of all the residue vectors representing integers in $\left[0, M_{I_{S}}\right)$.

Every codeword of an $\mathrm{RR}-(n, k, \alpha)$-code has an information part consisting of the first $k$ residue digits and a parity part, consisting of the remaining $n-k$ residue digits. Moreover, as proven in [9], $C$ is semilinear, i.e., it is linear under certain conditions. All the main properties of the linear codes also hold for the semilinear codes.

In particular,

Lemma 1. [9] An $R R$ - $(n, k)$-code $C$ has a minimum distance $d$ if and only if

$\max \left\{\Pi_{i=1}^{d} m_{j_{i}}\right\}>M_{R} \geq \max \left\{\Pi_{i=1}^{d-1} m_{j_{i}}\right\}$, for $1 \leq j_{i} \leq n$.

Therefore,

Lemma 2. [2], [9] For fixed $k \in[1, \ldots, n-1]$, the $R R$ - $(n, k)$-code $C$ defined by the moduli $m_{1}, \ldots, m_{k}$, $m_{k+1}, \ldots, m_{n}$ such that $m_{1}<m_{2}<\ldots<m_{k}<m_{k+1}<$ $\ldots<m_{n}$ has minimum distance $d=n-k+1$ and, hence, it is an MDS code. Moreover, varying the number $k$ of nonredundant moduli from 1 to $n-1$, we obtain $n-1$ different MDS codes.

Fact 1. Given $n$ moduli $m_{1}, \ldots, m_{k}, m_{k+1}, \ldots, m_{n}$ and a fixed integer $k \in[1, \ldots, n-1]$, let $M_{I_{S}}=\prod_{i=1}^{k} m_{i}$ and $M_{R}=\Pi_{i=k+1}^{n} m_{i}$. Then, the entire space $S=m_{1} \times m_{2} \times$ $\ldots m_{k} \times m_{k+1} \times \ldots \times m_{n}$ can be partitioned into $M_{R}$ subspaces, denoted as $C_{0}, C_{1}, \ldots C_{M_{R}-1}$ such that $C_{j}=R R-\left(n, k, j M_{I_{S}}\right)$.

\section{Disk Allocation Strategy Based on Redundant Residue Codes}

We are now ready to present the Decluster procedure, which solves the disk allocation problem for multidimensional files whose attributes have pairwise prime domains. For fixed $k \in[1, \ldots, n-1]$, the procedure Decluster in Fig. 1 distributes a file $F\left(m_{1}, \ldots, m_{k}, m_{k+1}, \ldots, m_{n}\right)$ among a set of $D=\Pi_{i=k+1}^{n} m_{i}$ disks denoted as $d_{0}, d_{1}, \ldots, d_{D-1}$, with the help of the $\operatorname{RR}-(n, k, 0)$-code $=\mathrm{C}_{0}=\left[\mathrm{n}, \Pi_{\mathrm{i}=1}^{\mathrm{k}} \mathrm{m}_{\mathrm{i}}, \mathrm{d}\right]$, whose codewords are the residue representations of the integers in the range $\left[0, \Pi_{i=1}^{k} m_{i}\right)$. In this paper, $C_{0}$ will be called the seed code.

Let us prove some properties of the above procedure.

Lemma 3. The Decluster procedure guarantees that every partial match query (PMQ) $q$ on the file $F$ with at most $d-1$ unspecified attributes has response time $R T(q)=1$.

Proof. No more than one bucket in each disk qualifies for $q$ because the seed code has minimum distance $d$ and, thus, two buckets stored in the same disk must differ in at least $d$ attributes. 


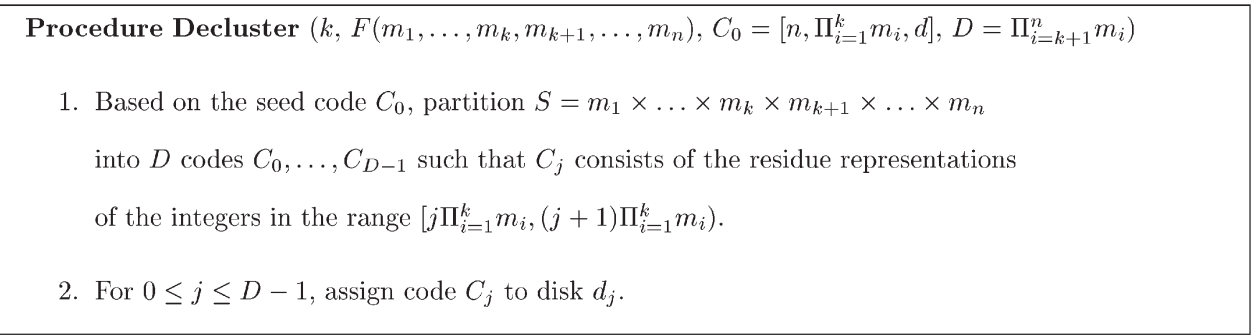

Fig. 1. The Decluster procedure.

Theorem 1. Let the pairwise-prime domains of the attributes of the file $F\left(m_{1}, \ldots, m_{k}, m_{k+1}, \ldots, m_{n}\right)$ be arranged in an increasing order such that

$$
m_{1}<\ldots<m_{k}<m_{k+1}<\ldots<m_{n} .
$$

For fixed $k \in[1 . . n-1]$, given the seed code

$$
C_{0}=\left[n, \Pi_{i=1}^{k} m_{i}, n-k+1\right],
$$

the associated Decluster procedure leads to a strictly optimal disk allocation strategy for every PMQ q on F. Hence, using $D=\Pi_{i=k+1}^{n} m_{i}$ disks, the response time for every PMQ $q$ whose response set has size $N(q)$ is given by $R T(q)=\left\lceil\frac{N(q)}{D}\right\rceil$.

Proof. Fixed $k$ : For any partial match query with at most $n-k$ unspecified attributes, the result follows from Lemma 3 and the fact that $N(q) \leq D$.

For PMQs with more than $n-k$ unspecified attributes, let $q$ be an arbitrary query whose attributes $q_{i_{1}}, \ldots, q_{i_{u}}$ are specified, and the remaining $n-u$ attributes satisfy $q_{i_{j}}=a_{i_{j}}$ for $u+1 \leq j \leq n$.

Let $M_{s}=\Pi_{j=u+1}^{n} m_{i_{j}}$ be the product of the domains of the unspecified attributes of $q$. By the Chinese Remainder Theorem [2], there is a unique integer $X$ in the range $\left[0, M_{s}\right)$ such that $X \equiv a_{i_{u+j}} \bmod m_{i_{u+j}}$, for $1 \leq j \leq n-u$. In the range $[0, M)$, where $M=\Pi_{i=1}^{n} m_{i}$, there are $\frac{M}{M_{s}}$ strings $\left.<x_{1}, \ldots, x_{n}\right\rangle$ such that $x_{i_{u+j}}=a_{i_{u+j}}$ for $1 \leq j \leq n-u$. Those $n$-tuples correspond to the integers $X+k M_{s}$ where $0 \leq k<\frac{M}{M_{s}}$, and verify $\left(X+k M_{s}\right) \equiv X \bmod m_{i_{u+j}}$ for $1 \leq j \leq n-u$. Recall that, by Fact 1 , the code $C_{j}$ associated with the disk $d_{j}$, for $0 \leq j \leq D-1$, consists of the residue representations of the integers in $\left[j \Pi_{i=1}^{k} m_{i},(j+1) \Pi_{i=1}^{k} m_{i}\right)$. There are at most $\left\lceil\frac{\Pi_{i=1}^{k} m_{i}}{M_{s}}\right\rceil$ buckets qualifying for $q$ among the codewords of $C_{j}$. Hence, the response time is $R T(q)=\left\lceil\frac{\Pi_{i=1}^{k} m_{i}}{M_{s}}\right\rceil$. Since $N(q)=\frac{M}{M_{s}}$ and $D=\Pi_{i=k+1}^{n} m_{i}$, for every PMQ $q$, the claim follows:

$$
R T(q)=\left\lceil\frac{\Pi_{i=1}^{k} m_{i}}{M_{s}}\right\rceil=\left\lceil\frac{M / D}{M_{s}}\right\rceil=\left\lceil\frac{M / M_{s}}{D}\right\rceil=\left\lceil\frac{N(q)}{D}\right\rceil .
$$

\subsection{Capturing Dynamic Scenario}

Our solution to the disk allocation problem can efficiently manage situations in which either 1) the number of disks available in the system changes, or 2) the number of attributes in a file changes, without redistributing the entire multidimensional file from scratch among the disks. Nonetheless, in either cases, some buckets must be moved from one disk to another.

Assume a pairwise-prime multidimensional file

$$
F\left(m_{1}, \ldots, m_{k}, m_{k+1}, \ldots, m_{n}\right)
$$

with

$$
m_{1}<\ldots<m_{k}<m_{k+1}<\ldots<m_{n},
$$

that has been distributed by the Decluster procedure among $D=\Pi_{i=k+1}^{n} m_{i}=M_{R}$ disks using the MDS seed code $C_{0}=\left[n, M_{I_{S}}=\Pi_{i=1}^{k} m_{i}, n-k+1\right]$.

First, suppose the number of available disks increases from $D$ to $m_{k} D$. To reduce the response time, fewer buckets will be assigned to each disk. The file $F$ will be declustered using the new MDS seed code $C^{+}=\left[n, \frac{M_{I_{s}}}{m_{k}}, n-k+2\right]$, which assigns $\frac{M_{I_{s}}}{m_{k}}$ buckets to each disks in the new configuration. This means that each code stored at the beginning into a single disk $d_{j}$, for $0 \leq j \leq M_{R}-1$, is now spread out among the $m_{k}$ disks,

$$
\left\{m_{k} d_{j}, m_{k} d_{j}+1, \ldots,\left(m_{k}+1\right) d_{j}-1\right\}
$$

Moreover, the new solution is strictly optimal because the new response time is $R T^{+}(q)=\left\lceil\frac{N(q)}{D m_{k}}\right\rceil$.

Similarly, if the number of available disks reduces from $M_{R}$ to $\frac{M_{R}}{m_{k+1}}$, a new MDS seed code $C^{-}=\left[n, M_{I} m_{k+1}, n-k\right]$ will be used to decluster $F$. The buckets distributed in $m_{k+1}$ consecutive disks in the initial disk system are now collapsed into a single disk of the new disk system. The new disk allocation remains strictly optimal. Note that the response time increases because fewer disks are available and only the PMQ with no more than $n-k-1$ unspecified attributes can now exhibit constant response time.

Finally, observe that when the number $(D)$ of disks available is not a multiple of the attribute domains, the fastest disk allocation strategy achievable with our strategy will involve $\bar{D}=\prod_{i=n-u+1}^{n} m_{i}$ disks where $\bar{D}$ is the product of the $u$ largest domains of $F$ such that $\Pi_{i=n-u}^{n} m_{i}>D \geq \Pi_{i=n-u+1}^{n} m_{i}$. For every PMQ $q$, the response time $R T(q)$ using only $\bar{D}$ disks is slower than the response time $R T^{*}(q)$ achieved using all the $D$ disks. However, the slowdown is upper bounded by $m_{n-u}$ because $R T(q)<R T^{*}(q) m_{n-u}$.

Suppose now that $D$ remains unchanged, while the number of attributes of the file $F$ changes. Although this 
situation may have less impact in practice, this could be the case when more refined searches, using a new attribute of $F$, are performed or a previously used attribute of the file becomes obsolete. If $F$ is searched according to a new attribute $n+1$, whose domain is $m_{n+1}$, each bucket $\left\langle a_{1}, \ldots, a_{n}\right\rangle$ is partitioned into the $m_{n+1}$ buckets. These are

$$
\begin{aligned}
& <a_{1}, \ldots, a_{n}, 0>,<a_{1}, \ldots, a_{n}, 1>, \\
& \ldots,<a_{1}, \ldots, a_{n}, m_{n+1}-1>,
\end{aligned}
$$

one for each value of the $(n+1)$ th attribute. The number of buckets increases from $M=\prod_{i=1}^{n} m_{i}$ to $M^{\prime}=M m_{n+1}$. Since the number of disks is unchanged, more buckets must be assigned to each disk. Assuming the MDS $C=\left[n, M_{I_{S}}, n-k+1\right]$, as the seed code according to which $F$ was distributed, the new seed code will be $C^{*}=$ $\left[n+1, M_{I_{S}} m_{n+1}, t\right]$ where $t \leq n-k+1$. Depending on the value of $m_{n+1}, C^{*}$ may or may not be an MDS code and, therefore, optimality is no longer guaranteed. About the relocation process, the bucket $\left\langle a_{1}, \ldots, a_{n}\right\rangle$ initially stored into the disk $d_{j}$ is now partitioned into $m_{n+1}$ buckets, as mentioned above. Precisely, the buckets $\left.<a_{1}, \ldots, a_{n}, x_{n+1}\right\rangle$, which is the residue representation of $X+k M$ for fixed $k \in\left[0, m_{n+1}-1\right]$, will be assigned to

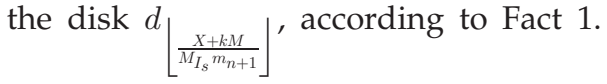

A similar reasoning applies when the number of attributes of $F$ decreases.

\section{Redundant Nonpairwise Prime REsidue Codes}

The solution proposed in the last section works for pairwise-prime files. This is not a strong constraint because the domains of the attributes are selected while the database is designed depending on the original interval of the attributes and on the size of the disk page (see Example 2). Nevertheless, for the sake of completeness, let us extend our solution to the case of nonpairwise prime domains.

Consider a set of $n$ nonpairwise prime moduli $m_{1}, m_{2}, \ldots, m_{k}, m_{k+1}, \ldots, m_{n}$ and also consider all the $n$-tuples $\left\langle x_{1}, \ldots, x_{n}\right\rangle$ for $x_{i} \in\left[0, m_{i}\right)$, of the $n$-dimensional space $S=m_{1} \times \ldots \times m_{n}$. Let $M=\Pi_{i=1}^{n} m_{i}$ and $\tau=$ l.c.m. $\left(m_{1}, m_{2}, \ldots, m_{n}\right)$ be, respectively, the product and the least common multiple (1.c.m.) of the $n$ moduli.

In contrast to the pairwise-prime case, there are $n$-tuples $\left.<x_{1}, \ldots, x_{n}\right\rangle \in S$ which do not represent any integer $X$. That is, there is no integer $X$ such that $x_{i} \equiv X \bmod m_{i}$, for $1 \leq i \leq n$. In fact, a general form of the Chinese Remainder Theorem [11] implies that, if $S$ consists of $M$ strings, only the integers in $[0, \tau)$ have a residue representation $<x_{1}, \ldots, x_{n}>\in S$. Precisely, if $g_{i j}=$ g.c.d $\left(m_{i}, m_{j}\right)$ is the greatest common divisor (g.c.d.) of the two moduli $m_{i}$ and $m_{j}$, only those $n$-tuples which satisfy the congruence

$$
x_{i} \equiv x_{j} \bmod g_{i j}, \text { for } 1 \leq i, j \leq n
$$

are valid integer representations in the $n$-dimensional space $S$.

Note that those $\tau$ valid residue representations form a subset of $S$, which is a code by definition. However, the minimum distance of such a code is unknown (if the code itself is not generated). To overcome this limitation, some explicit redundancy can be added as follows.

\section{Definition 5. Given $n$ nonpairwise prime moduli}

$$
m_{1}, \ldots, m_{k}, m_{k+1}, \ldots, m_{n}
$$

for fixed $k \in[1, \ldots, n-1]$, let the first $k$ moduli be called nonredundant and the remaining $n-k$ moduli be called redundant. Moreover, let $\Lambda=1 . \operatorname{c.m}\left(m_{1}, m_{2}, \ldots, m_{k}\right)$ and $\tau=1 . \operatorname{c.m}\left(m_{1}, m_{2}, \ldots, m_{n}\right)$. The nonpairwise prime redundant residue code or NPRR-( $n, k)$-code $C$ consists of the set of valid integer representations, i.e., the set of representations of all integers in $[0, \Lambda)$.

It is easy to see that the $\operatorname{NPRR}-(n, k)$-code $C$ is a semilinear code. To derive the minimum distance of the NPRR- $(n, k)$-code $C$, let

- $\quad \wp$ denote the l.c.m. of the $n-k$ redundant moduli;

- $\quad \Lambda$ denote the l.c.m. of the $k$ nonredundant moduli;

- $\quad \tau$ denote the l.c.m. of all $n$ moduli;

- $\quad$ partitioning the $n$ moduli into two arbitrary sets $s_{d-1}$ and $s \overline{d-1}$ of sizes $d-1$ and $n-d+1$ respectively, let $\mu_{s_{d-1}}$ and $\mu_{s \overline{d-1}}$ be the 1.c.m of the moduli in $s_{d-1}$ and $s \overline{d-1}$, respectively. In other words, $\mu_{s_{d-1}}=$ l.c.m. $\left(m_{i_{j}} \mid m_{i_{j}} \in s_{d-1}\right)$ and

$$
\mu_{s_{\overline{d-1}}}=\text { l.c.m. }\left(m_{i_{j}} \mid m_{i_{j}} \in s \overline{d-1}\right) ;
$$

- $\quad$ partitioning the $n$ moduli in two arbitrary sets $s_{d}$ and $s_{\bar{d}}$ of sizes $d$ and $n-d$, respectively, let $\nu_{s_{d}}$ and $\nu_{s_{\bar{d}}}$ be the l.c.m of the moduli in $s_{d}$ and $s_{\bar{d}}$, respectively. That is, $\nu_{s_{d}}=$ l.c.m. $\left(m_{i_{j}} \mid m_{i_{j}} \in s_{d}\right)$ and

$$
\nu_{s_{\bar{d}}}=\text { l.c.m. }\left(m_{i_{j}} \mid m_{i_{j}} \in s_{\bar{d}}\right) \text {; }
$$

- $\mathcal{S}_{d}$ be the set of all possible $s_{d}$ subsets among $\left\{m_{1}, \ldots, m_{n}\right\}$;

- $\mathcal{S}_{d-1}$ be the set of all possible $s_{d-1}$ subsets among $\left\{m_{1}, \ldots, m_{n}\right\}$.

Theorem 2. Given the radices $m_{1}, \ldots, m_{n}$ for fixed $k \in$ $[1 . . n-1]$, the NPRR-(n,k)-code $C$ has the minimum distance $d$ if and only if the following relation holds:

$$
\begin{aligned}
& \max _{s_{d} \in \mathcal{S}_{d}}\left(\frac{\nu_{s_{d}}}{\operatorname{gcd}\left(\nu_{s_{d}}, \nu_{s_{\bar{d}}}\right)}\right)>\frac{\wp}{\operatorname{gcd}(\Lambda, \wp)} \\
& \geq \max _{s_{d-1} \in \mathcal{S}_{d-1}}\left(\frac{\mu_{s_{d-1}}}{\operatorname{gcd}\left(\mu_{s_{d-1}}, \mu_{s_{\overline{d-1}}}\right)}\right) .
\end{aligned}
$$

Proof. The semilinear NPRR- $(n, k)$-code $C$ has minimum distance $d$ if no codeword has Hamming weight $\leq$ $d-1$ (except for the all-zero codeword) and if there is at least one codeword of Hamming weight $d$. A codeword with $d-1$ nonzero digits represents an integer $X=X^{\prime} \mu_{s_{d-1}}$, for some $s_{d-1}$. The smallest value of $X$ is obtained for $X^{\prime}=1$ and $\min _{s_{d-1} \in \mathcal{S}_{d-1}}\left(\mu_{s_{d-1}}\right)$. 
Procedure Partition $\left(S=m_{1} \times \ldots \times m_{n}, C_{0}\right)$

1. Build $\frac{\tau}{\Lambda}$ codes $C_{0}, C_{1}, \ldots, C_{\frac{\tau}{\Lambda}-1}$ as defined in Fact 2, and let $Z=C_{0} \cup C_{1} \cup \ldots \cup C_{\frac{\tau}{\Lambda}-1}$.

2. From the remaining $(M-\tau) n$-tuples of the space $S=m_{1} \times \ldots \times m_{n}$, find a set of $\frac{M}{\tau} n$-tuples $T=\left\{T(0), T(1), \ldots, T\left(\frac{M}{\tau}-1\right)\right\}$ such that

- $T(0)$ is the all-zero codeword;

- for every pair $i, j$, with $1 \leq i<j<\frac{M}{\tau}, T(i)-T(j) \notin Z$ or $T(j)-T(i) \notin Z$.

3. Compute the multiple-sum between each code $C_{0}, C_{1}, \ldots, C_{\frac{\tau}{\Lambda}-1}$ and each $n$-tuple of $T$. That is, compute the $M / \Lambda$ sets $C_{j}[T(i)]$, where $0 \leq j \leq \tau / \Lambda-1$ and $0 \leq i \leq M / \tau-1$.

Fig. 2. The Partition procedure.

Consequently, the corresponding word is not a codeword if and only if

$$
\min _{s_{d-1} \in \mathcal{S}_{d-1}}\left(\mu_{s_{d-1}}\right) \geq \Lambda
$$

or equivalently

$$
\left.\frac{\tau}{\max _{s_{d-1} \in \mathcal{S}_{d-1}}\left(\frac{\mu_{s_{d-1}}}{\operatorname{gcd}\left(\mu_{s_{d-1}}, \mu_{s_{d-1}}\right.}\right)}\right) \geq \Lambda,
$$

where

$$
\tau=\frac{\mu_{s_{d-1}} \mu_{s_{\overline{d-1}}}}{\operatorname{gcd}\left(\mu_{s_{d-1}}, \mu_{s_{\overline{d-1}}}\right)} .
$$

Now, if a codeword of Hamming weight $d$ exists, the above condition must be denied for any subset of $d$ digits. Hence,

$$
\frac{\tau}{\max _{s_{d} \in \mathcal{S}_{d}}\left(\frac{\nu_{s_{d}}}{\operatorname{gcd}\left(\nu_{s_{d}}, \nu_{s_{d}}\right)}\right)}<\Lambda .
$$

From the above two conditions and recalling that $\tau$ can be expressed as $\tau=\frac{\Lambda_{\wp}}{\operatorname{gcd}(\Lambda, \wp)}$, the claim follows.

For nonpairwise prime redundant residue codes, Fact 1 can be rewritten as follows:

Fact 2. For fixed $k \in[1, \ldots, n-1]$ and given a set of $n$ nonpairwise prime radices $m_{1}, \ldots, m_{n}$, let $m_{1}, \ldots, m_{k}$ and $m_{k+1}, \ldots, m_{n}$ be, respectively, the nonredundant and the redundant moduli. Considering the $\operatorname{NPRR}-(n, k)$-code $C=[n, \Lambda, d]$, all the integers in $[0, \tau)$, can be partitioned into $\frac{\tau}{\Lambda}$ codes, each having the same size and the same distance as $C$. More precisely, code $C_{j}$ for $0 \leq j \leq \frac{\tau}{\Lambda}-1$, consists of the set of residue representations of the integers $X \in[j \Lambda,(j+1) \Lambda-1]$. Note that $C_{0}=C$.

Proof. The proof follows directly by observing that the Hamming distance of the two codewords $x_{1}$ and $x_{2}$ belonging to $C_{0}$ is the same as the Hamming distance between the two codewords $x_{1}+j \Lambda$ and $x_{2}+j \Lambda$ belonging to $C_{j}$, where $0 \leq j \leq \frac{\tau}{\Lambda}-1$.
Finally, let the multiple-sum set, $C[x]=\{x+w \mid w \in C\}$, be obtained by the modular sum, digit-by-digit, of the $n$-tuple $x$ over all the $n$-tuples of the code $C$.

Now, for a given NPRR- $(n, k)$-code $C_{0}$, the entire $n$-dimensional space $S=m_{1} \times \ldots \times m_{n}$ can be partitioned by the algorithm in Fig. 2 .

To prove the correctness of the above procedure, let us prove the following:

Lemma 4. The set of subsets $\left\{C_{j}[T(i)] \mid 0 \leq j \leq \tau / \Lambda-1\right.$ and $0 \leq i \leq M / \tau-1\}$ forms a partition of the space $S=$ $m_{1} \times \ldots \times m_{n}$. Moreover, each subset $C_{j}[T(i)]$ is a code $[n, \Lambda, d]$.

Proof. First note that $C_{j}[T(0)]=C_{j}$, for $0 \leq j \leq \tau / \Lambda-1$. Next, let us prove, by contradiction, that $C_{j}[T(i)] \cap$ $C_{r}[T(s)]=\emptyset$ for any choice of $i, j, r, s$. Suppose that the two $n$-tuples $X(j, i) \in C_{j}[T(i)]$ and $Y(r, s) \in C_{r}[T(s)]$, obtained by the residue vectors $X, Y \in C_{0}$, are equal. W.l.o.g. assume $j \geq r$. Note that $X(j, i)$ is the sum of the residue representation of $(X+j \Lambda)$ and of the $n$-tuple $T(i)$, while $Y(r, s)$ is the sum of the residue representation of $(Y+r \Lambda)$ and of the $n$-tuple $T(s)$. Now, $X(j, i)$ is equal to $Y(r, s)$ if and only if $X-Y+$ $(j-r) \lambda=T(s)-T(i)$ belongs to $C_{j-r}$. This cannot be true since it is against the criteria used for the selection of the $n$-tuples in $T$. Hence, the subsets in $\left\{C_{j}[T(i)] \mid\right.$ $0 \leq j \leq \tau / \Lambda-1$ and $0 \leq i \leq M / \tau-1\}$ form a partition of the space $S=m_{1} \times \ldots \times m_{n}$.

It still remains to be proven that, for any pair $j, i$, the set of subsets $C_{j}[T(i)]$ has the same minimum distance as $C_{0}$. Consider two arbitrary $n$-tuples $X(j, i)$ and $Y(j, i)$, both belonging to $C_{j}[T(i)]$, and derived from $X$ and $Y$ in $C_{0}$. Repeating the above reasoning, it is easy to see that the $n$-tuples $X(j, i)-Y(j, i)$ and $X-Y$ are the same. Hence, all the subsets in $\left\{C_{j}[T(i)] \mid 0 \leq j \leq \tau / \Lambda-1\right.$ and $0 \leq i \leq M / \tau-1\}$ inherit the same minimum distance $d$ as the set $C_{0}$.

Finally, the decluster procedure for nonpairwise primes is described in Fig. 3. 


$$
\begin{aligned}
& \text { Procedure NonPairwise Prime Decluster } \\
& \qquad\left(k, F\left(m_{1}, \ldots, m_{k}, m_{k+1}, \ldots, m_{n}\right), C_{0}=[n, \Lambda, d], D=\frac{M}{\Lambda}\right) \\
& \text { 1. Partition }\left(S=m_{1} \times \ldots \times m_{n}, C_{0}\right) ; \\
& \quad / * \text { This step builds the } D \operatorname{codes}\left\{C_{j}[T(i)] \mid 0 \leq j \leq \tau / \Lambda-1 \text { and } 0 \leq i \leq M / \tau-1\right\} . * / \\
& \text { 2. For } 0 \leq j \leq \tau / \Lambda-1 \text { and } 0 \leq i \leq M / \tau-1 \text {, assign code } C_{j}[T(i)] \text { to the disk } d_{i \frac{\tau}{\Lambda}+j .} .
\end{aligned}
$$

Fig. 3. The Nonpairwise Prime Decluster procedure.

Theorem 3. For a fixed $k \in[1 . . n-1]$, let

$$
F\left(m_{1}, \ldots, m_{k}, m_{k+1}, \ldots, m_{n}\right)
$$

be a file whose attributes are not pairwise prime and let $C_{0}=$ $[n, \Lambda, d]$ be the seed code. Distributing $F$ among $D=\frac{M}{\Lambda}$ disks using the Nonpairwise Prime Decluster procedure, the response time for a PMQ $q$ with $s$ specified attributes $q_{i_{1}}, \ldots, q_{i_{s}}$ is given by $R T(q)=\left\lceil\frac{\Lambda}{M_{s}}\right\rceil$, where

$$
M_{s}=1 . \operatorname{com}\left(m_{i_{1}}, \ldots, m_{i_{s}}\right) .
$$

Finally, the ratio between the response time of this procedure, say $R T(q)$, and the best achievable response time, say $R T^{*}(q)$, is given by

$$
\left\lceil\frac{R T(q)}{R T^{*}(q)}\right\rceil=\left\lceil\frac{\left\lceil\frac{\Lambda}{M_{s}}\right\rceil}{\left\lceil\frac{\Lambda}{\Pi_{j=1}^{s} m_{i_{j}}}\right\rceil}\right\rceil .
$$

Proof. Consider the $n$-tuple $q^{\prime}$ belonging to $S=m_{1} \times m_{2} \times$ $\ldots \times m_{n}$ and obtained by substituting all the unspecified attributes of the PMQ $q$ with 0 . By the previous discussion, there must be $i$ and $j$ such that $q^{\prime} \in C_{j}[T(i)]$, and $q^{\prime}$ is stored into disk $d_{i \frac{\tau}{\Lambda}+j}$ and there exists $x \in C_{0}$ such that $q^{\prime}=x+j \Lambda+T(i)$. Then, $d_{i \frac{\tau}{\Lambda}+j}$ stores $q^{\prime}$ along with the buckets $q^{\prime}+u M_{s}=x+j \Lambda+T(i)+u M_{s}$, for all values of $u$ such that $0 \leq\left(x+u M_{s}\right) \leq \Lambda$. Note that $u M_{s}$ has always a valid residue presentation since $u M_{s}<\tau$. Therefore, at most $\left\lceil\frac{\Lambda}{M_{s}}\right\rceil$ buckets qualify for the query $q$ on the same disk.

It remains to show how far is the response time for nonpairwise prime decluster procedure from the optimal response time. Using $D=\frac{M}{\Lambda}$ disks, the optimal response time for $q$ would be

$$
R T^{*}(q)=\left\lceil\frac{N(q)}{D}\right\rceil=\left\lceil\frac{\overline{\Pi_{j=1}^{s} m_{i_{j}}}}{\frac{M}{\Lambda}}\right\rceil=\left\lceil\frac{\Lambda}{\Pi_{j=1}^{s} m_{i_{j}}}\right\rceil .
$$

Therefore, our solution is

$$
\left\lceil\frac{\left\lceil\frac{\Lambda}{M_{s}}\right\rceil}{\left\lceil\frac{\Lambda}{\Pi_{j=1}^{s} m_{i_{j}}}\right\rceil}\right\rceil
$$

times slower than the strictly optimal solution.
Example 3. Consider the redundant residue number system (RNS) associated with the nonpairwise prime radices, $m_{1}=3, m_{2}=6, m_{3}=5$. For fixed $k=2$, let $m_{1}, m_{2}$ be the nonredundant moduli and let $m_{3}$ be the redundant modulus. According to our definitions, $M=90, \tau=30$, and $\Lambda=6$. In other words, the space $S$ consists of $3 \times$ $6 \times 5$ number of 3 -tuples, but the RNSs associated with the moduli $m_{1}, m_{2}, m_{3}$ and $m_{1}, m_{2}$, can represent the integers in $[0,29]$ and $[0,5]$, respectively. Let the code $C=[3,6,2]$ consist of the residue representations of the integers in $[0,5]$ in the RNS of radices $m_{1}=3, m_{2}=6$, $m_{3}=5$. That is,

$$
\begin{aligned}
C=\{ & <0,0,0>,<1,1,1>,<2,2,2>, \\
& <0,3,3>,<1,4,4>,<2,5,0>\} .
\end{aligned}
$$

From Fact 2, the valid residue representations are partitioned into $\frac{90}{6}=15$ codes. Precisely, code $C_{j}[0,0,0]$ corresponds to the residue representations of the integers in the range $[6 j, 6(j+1)-1]$, where $0 \leq j \leq 4$. In particular,

$$
\begin{aligned}
C_{1}=\{ & <0,0,1>,<1,1,2>,<2,2,3> \\
& <0,3,4>,<1,4,0>,<2,5,1>\}, \\
C_{2}=\{ & <0,0,2>,<1,1,3>,<2,2,4> \\
& <0,3,0>,<1,4,1>,<2,5,2>\}, \\
C_{3}=\{ & <0,0,3>,<1,1,4>,<2,2,0>, \\
& <0,3,1>,<1,4,2>,<2,5,3>\}, \text { and } \\
C_{4}=\{ & <0,0,4>,<1,1,0>,<2,2,1>, \\
& <0,3,2>,<1,4,3>,<2,5,4>\} .
\end{aligned}
$$

Now, let $T=\{T(0)=<0,0,0>, T(1)=<1,0,3>, T(2)=$ $<2,3,0\rangle\}$ be the set of 3 -tuples such that $T(1), T(2)$, $T(1)-T(2)=<2,3,3>$, and $T(2)-T(1)=<1,3,2>$ does not belong to $Z=C_{0} \cup C_{1} \cup C_{2} \cup C_{3} \cup C_{4}$. Hence, the codes $\left.C_{j}[1,0,3]=\{<1,0,3\rangle+C_{j}[0,0,0]\right\}$ and

$$
C_{j}[2,3,0]=\left\{<2,3,0>+C_{j}[0,0,0]\right\},
$$

for $0 \leq j \leq 4$, complete the partition of $S$.

Finally, the nonpairwise prime decluster procedure assigns the code $C_{j}[T(i)]$ to the disk $d_{5 i+j}$. Altogether there are 15 codes and hence $D=15$ disks are required.

Now, consider the PMQ $q=<*, *, 2>$. Since $M_{s}=5$, there are at most $\left\lceil\frac{\Lambda}{M_{s}}\right\rceil=2$ qualifying buckets for each disk. Hence, $R T(q)=2=R T^{*}(q)$ in this case. 


\section{Conclusion}

In this paper, we studied the disk allocation problem for distributing nonuniform multidimensional files on to parallel database systems that exploit the ability of accessing multiple disks simultaneously. Based on a large and flexible class of maximum distance separable codes, called the redundant residue codes, a strictly optimal allocation method is derived for every query $q$ when the attribute domains of the multidimensional file are pairwise prime. We also introduced a new family of residue codes, called the redundant nonpairwise prime residue codes, that can be applied with multidimensional files that have attribute domains nonpairwise prime.

\section{ACKNOWLEDGMENTS}

This work is partially supported by the Texas Advanced Research Program TARP-003594013 and a MIUR REALWINE research grant in Italy. A preliminary version of this paper appeared in [4]. C.M. Pinotti is grateful to Professor Barsi for introducing her to the area of the redundant residue number systems. The authors would also like to thank the reviewers for helpful suggestions and the associate editor for timely handling the paper.

\section{REFERENCES}

[1] K.A.S. Abdel-Ghaffar and A. El Abbadi, "Optimal Disk Allocation for Partial Match Queries," ACM Trans. Database Systems, vol. 18, no. 1, pp. 132-156, Mar. 1993.

[2] F. Barsi and P. Maestrini, "Error Correcting Properties of Redundant Residue Number Systems," IEEE Trans. Computers, vol. 22, pp. 307-315, 1973.

[3] F. Barsi and P. Maestrini, "Error Codes in Residue Number Systems with Non-Pairwise-Prime Moduli," Information and Control, vol. 46, no. 1, pp. 16-25, July 1980.

[4] S.K. Das and M.C. Pinotti, "An Optimal Disk Allocation Strategy for Partial Match Query of Non-Uniform Cartesian Product Files," Proc. IEEE Int'l Parallel Processing Symp., pp. 550-554, Apr. 1999.

[5] H.C. Du and J.S. Sobolewski, "Disk Allocation for Cartesian Product Files," ACM Trans. Database Systems, vol. 7, no. 1, pp. 82101, Mar. 1982.

[6] M.F. Fang, R.C.T. Lee, and C.C. Chang, "The Idea of DeClustering and its Applications," Proc. 12th Int'l Conf. Very Large Databases, pp. 181-188, Aug. 1986.

[7] C. Faloutsos and D. Metaxas, "Disk Allocation Methods Using Error Correcting Codes," IEEE Trans. Computers, vol. 40, no. 8, pp. 907-913, Aug. 1991.

[8] M.H. Kim and S. Pramanik, "Optimal File Distribution for Partial Match Retrieval," Proc. ACM-SIGMOD Int'l Conf. Management of Data, pp. 173-182, 1988.

[9] H. Khrisna, K. Lin, and J. Sun, "A Coding Theory Approach to Error Control in Redundant Residue Number Systems-Part I: Theory and Single Correction," IEEE Trans. Circuits and Systems, II, vol. 39, no. 1, pp. 8-17, Jan. 1992.

[10] F.J. MacWilliams and N.J.A. Sloane, The Theory of Error-Correcting Codes, Parts I and II. New York: North-Holland, 1977.

[11] O. Ore, "The General Chinese Remainder Theorem," Am. Math. Monthly, pp. 365-370, 1952.

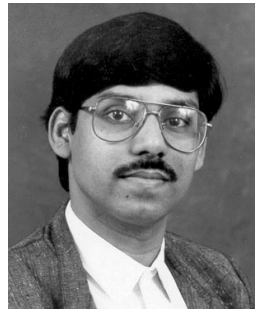

Sajal K. Das received the $\mathrm{PhD}$ degree in Computer Science in 1988 from the University of Central Florida, Orlando. Currently, he is a full professor of computer science and engineering and also the Founding Director of the Center for Research in Wireless Mobility and Networking (CReWMaN) at the University of Texas at Arlington (UTA). Prior to 1999, he was a professor of computer science at the University of North Texas (UNT), Denton, where he founded the Center for Research in Wireless Computing (CReW) in 1997, and also served as the director of the Center for Research in Parallel and Distributed Computing (CRPDC) from 1995-1997. He is a recipient of the UNT Student Association's Honor Professor Award in 1991 and 1997 for best teaching and scholarly research; UNT's Developing Scholars Award in 1996 for outstanding research; and UTA's Outstanding Senior Faculty Research Award in Computer Science in 2001. He has visited numerous universities, research organizations, and industry research labs for collaborative research and invited seminar talks. He was a visiting scientist at the Council of National Research in Pisa, Italy, and Slovak Academy of Sciences in Bratislava, and was also a Visiting Professor at the Indian Statistical Institute, Calcutta. His current research interests include resource and mobility management in wireless networks, mobile computing, QoS provisioning and wireless multimedia, mobile Internet, network architectures and protocols, distributed/parallel processing, performance modeling, and simulation. He has published more than 185 research papers in these areas, directed several projects funded by industry and government, and filed four US patents in wireless moble networks. He received the best paper awards for significant research contributions in the ACM Fifth International Conference on Mobile Computing and Networking (MobiCom'99), Third ACM International Workshop on Modeling, Analysis, and Simulation of Wireless and Mobile Systems (MSWiM 2000), and ACM/IEEE International Workshop on Parallel and Distributed Simulation (PADS'97). Dr. Das serves on the editorial boards of the Journal of Parallel and Distributed Computing, Parallel Processing Letters, Journal of Parallel Algorithms and Applications, and the Computer Networks journal. Each year he serves on numerous IEEE and ACM conferences as a technical program committee member, program chair, or general chair. He is a member of the IEEE TCPP Executive Committee and Advisory Boards of several cutting-edge companies. He is a member of the IEEE.

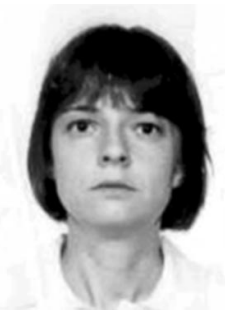

Cristina M. Pinotti received the PhD degree (cum laude) in computer science from the University of Pisa, Italy, in 1986. Since 2000, she has been an associate professor in the Department of Computer Science and Telecommunications of the University of Trento, Italy. Prior to 2000, she was a researcher with the Consiglio Nazionale delle Ricerche at the Istituto di Elaborazione dell'Informazione, Pisa. She was a visiting researcher at the Department of Computer Science, University of North Texas, Denton, and at the Department of Computer Science, Old Dominion University, Norfolk, Virginia. Her research interests include parallel data structures and multiprocessor interconnection networks, the design and analysis of parallel algorithms, I/O external memories, mobile and wireless networks, VLSI layouts, computer arithmetic, and residue number systems. Each year she serves on the program committee of several IEEE and ACM workshops and conferences. She is a member of the IEEE Computer Society.

$\triangleright$ For more information on this or any computing topic, please visit our Digital Library at http://computer.org/publications/dlib. 